\title{
Brillouin optical time-domain analyzer with a fiber ring laser working on the SLM regime
}

\author{
R. Ruiz-Lombera*a, L. Rodriguez-Cobo ${ }^{\mathrm{a}, \mathrm{b}}$, J. Mirapeix ${ }^{\mathrm{a}, \mathrm{b}}$, J.M. Lopez-Higuera ${ }^{\mathrm{a}, \mathrm{b}}$ \\ aphotonics Engineering Group, University of Cantabria, 39005, Santander (Spain) \\ ${ }^{\mathrm{b}}$ CIBER-bbn, Instituto de Salud Carlos III, 28029, Madrid (Spain)
}

\begin{abstract}
In this paper we present the employment of an Erbium Fiber Ring Laser structure working on the Single Longitudinal Mode regime within a Brillouin optical time domain analyzer. An analysis of some key laser parameters will be carried out, proving that a very stable operation is achieved. The associated performance of the BOTDA system, as well as the possible benefits derived from the use of the proposed laser design will also be discussed.
\end{abstract}

Keywords: BOTDA, stimulated Brillouin scattering, ring laser, SLM regime, noise sources

\section{INTRODUCTION}

Within the field of optical fiber sensors, distributed systems based on different scattering phenomena have been a very active area of research during the last years. In particular, Brillouin-based solutions have been widely explored to give rise to different solutions exploiting both spontaneous (SpBS) and stimulated (SBS) Brillouin scatterings. Among the latter, Brillouin optical time-domain analyzers (BOTDAs) have been the most commonly employed approach due to their ability to measure both temperature and strain with setups less complicated than those associated with other approaches such as Brillouin optical correlation or frequency analyzers (BOCDA and BOFDA, respectively).

The performance of BOTDAs has been improved with contributions in different areas: from the increase in the sensing length, for example via Raman ${ }^{1}$ or Brillouin assistance ${ }^{2}$, to the achievement of higher spatial resolutions ${ }^{3}$ or the ability to perform dynamic measurements ${ }^{4}$. In fact, a figure-of-merit (FoM) has been proposed ${ }^{5}$, enabling a tool to compare the performance of different BOTDA implementations. In this work ${ }^{5}$, an expression relating the error in the estimation of the Brillouin frequency shift (BFS) to the Brillouin linewidth and the signal-to-noise (SNR) ratio is also provided. The frequency error, and therefore the strain/temperature resolution, is inversely proportional to the SNR, thus indicating the impact of noise on the BOTDA performance.

Different authors have recently contributed to analyze the impact of different noise sources in BOTDAs. Urricelqui et al. ${ }^{6}$ studied and modeled the different BOTDA noise sources, concluding that the sensing range determines whether phase-to-intensity noise conversion in the SBS process and SpBS-probe beating noise (for short distances) or thermal and double Rayleigh scattering (DRS)-induced noise (generated by the probe wave) (for long distances) are the dominating effects. The effect of laser phase noise has been particularly analyzed in several works, considering both pump and probe as continuous waves ${ }^{7}$ and analyzing the influence of the laser linewidth on the noise system for different fiber lengths and pump pulse durations ${ }^{8}$.

Within this framework, we propose the use of a stable configuration of Erbium Fiber Ring Laser (EFRL) based on a uniform FBG working on the single longitudinal mode regime (SLM) as source of a BOTDA system. We will analyze the laser features, showing that some typical fiber ring laser problems such as multimode operation and stability are overcome by the proposed design. The inherent ability of FBG-based EFRLs to be tuned over a wide spectral range and to show very narrow linewidths might be of great interest for BOTDA implementations.

*ruben.ruiz@unican.es; phone (+34) 942 206736; gif.teisa.unican.es

25th International Conference on Optical Fiber Sensors, edited by Youngjoo Chung, Wei Jin,

Byoungho Lee, John Canning, Kentaro Nakamura, Libo Yuan, Proc. of SPIE Vol. 10323,

1032381 - (C) 2017 SPIE - CCC code: 0277-786X/17/\$18 - doi: 10.1117/12.2265443 


\section{EXPERIMENTAL ISSUES}

\subsection{Experimental setup}

An Erbium Fiber Ring Laser (EFRL) configuration based on commercial Er-doped fiber (I25 from Fibercore) has been employed as the laser source of a BOTDA sensor. Employing a uniform FBG as filter, the signal is launched through a non-pumped Er-doped fiber acting as saturable absorber to narrow the FBG response due to the Spatial Hole Burning $(\mathrm{SHB})^{9}$ and reach the Single Longitudinal Mode regime.

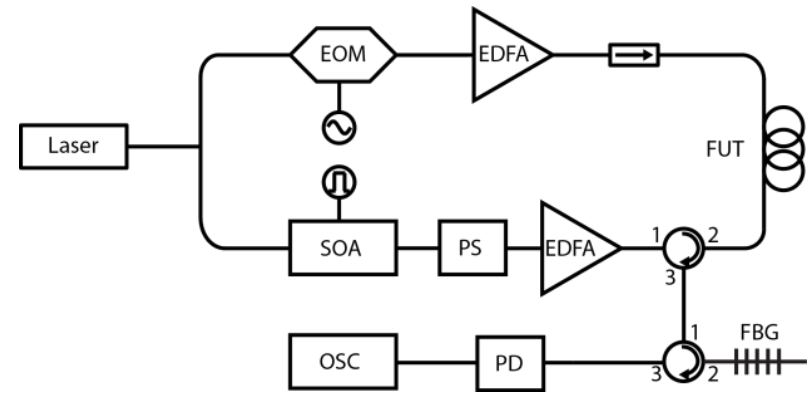

a)

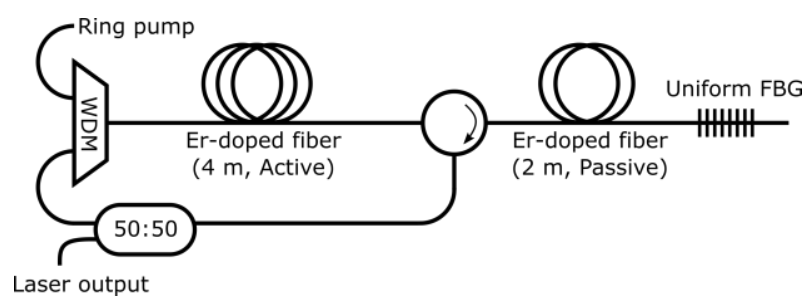

b)

Figure 1. Experimental setups. a) Standard BOTDA sensor system. EOM: electro-optic modulator; EDFA: Erbium doped fiber amplifier; SOA: semiconductor optical amplifier; PS: polarization scrambler; PD: photodetector; OSC: oscilloscope; FBG: fiber Bragg grating. b) Fiber Ring Laser configuration employed to achieve a SLM output. A section of Er-doped fiber is introduced before the FBG as saturable absorber to narrow the FBG response.

To validate the possibility of using an ERFL as a laser source for distributed Brillouin measurements, a standard BOTDA configuration was employed. The laser source emitting at around $1550 \mathrm{~nm}$ is divided equally into two optical paths associated with the pump and probe waves. The latter is modulated with an electro-optical modulator (EOM) and a $\mathrm{RF}$ generator using the double-sideband suppressed-carrier technique to generate the two sidebands. This wave is amplified using an Erbium-doped fiber amplifier (EDFA) to achieve a constant power of $500 \mu \mathrm{W}$ for each sideband and it is then launched into the fiber under test (FUT) of approximately $21 \mathrm{~km}$. The pump wave is pulsed using a semiconductor optical amplifier (SOA) with a 20ns pulse duration and $2 \mathrm{KHz}$ of repetition rate. It goes then through a polarization scramble to avoid the polarization dependence of the Brillouin gain. Then the pulse is amplified up to around $350 \mathrm{~mW}$ of peak power using an EDFA before entering the FUT. Finally, the probe wave is circulated to a fiber Bragg grating (FBG) where the lower sideband of the signal is reflected and then detected using a $125 \mathrm{MHz}$ photodetector and an acquisition card. The received signal is averaged 4096 times to improve the signal to noise ratio (SNR).

\section{RESULTS}

In order to check the SLM working regime of the ERFL employed, some key laser parameters have been measured, as the optical output spectra and the wavelength stability after some minutes. These parameters have been compared with the results obtained using a commercial DFB laser diode manufactured by EMCORE, with both lasers working around $1550 \mathrm{~nm}$. The ERFL structure presents the advantage that the operating wavelength could be easily modifying between 1500 to $1600 \mathrm{~nm}$ only by changing the chosen FBG.

Figure 2 presents spectral comparisons of both lasers using a Brillouin optical spectrum analyzer (BOSA) with a high spectral resolution of $0.082 \mathrm{pm}$. In order to equalize the output power of both lasers, an optical attenuator has been employed with the DFB to reduce the optical power down to $0 \mathrm{dBm}$ for a better comparison. In Figure 2 (a) the results obtained for both lasers show that the ERFL (red line) is slightly narrower than the DFB laser diode (blue line). As explained in ${ }^{8}$, the phase noise of the laser is directly proportional to its linewidth, and the noise of a BOTDA sensor increases linearly with the power spectral density of the laser frequency noise, so a narrower laser source could enable an 
improved performance. Figure 2 (b) shows the wavelength stability of both lasers after 10 minutes. The results present a similar behavior for both DFB and ERFL lasers, of around $\pm 1 \mathrm{pm}$ fluctuation with respect to the center wavelength. In BOTDA sensors, it is fundamental to be sure of using a fixed and stable operating wavelength. In the proposed standard implementation, just before the photodetector employed to detect the optical signal, the probe wave goes through a FBG and only one sideband must be reflected to the detector, so a wavelength consistency must be guaranteed between the laser source and the FBG.

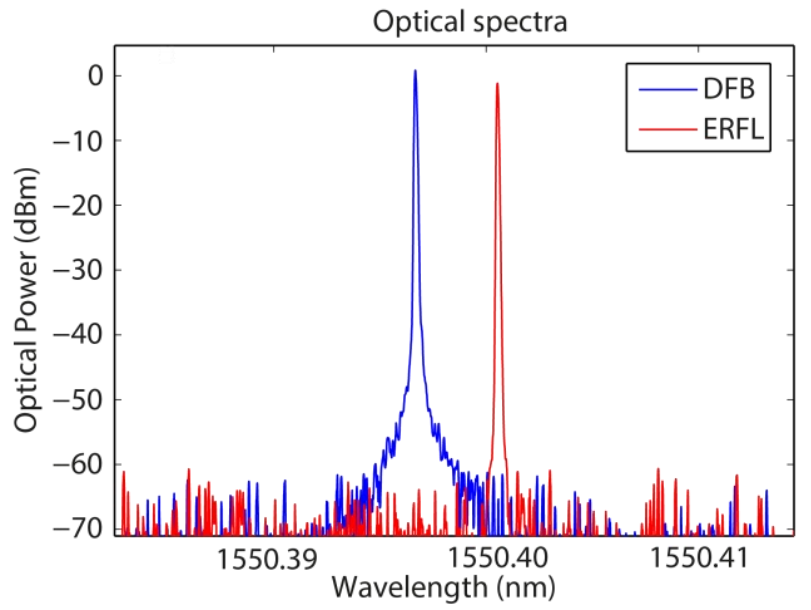

a)

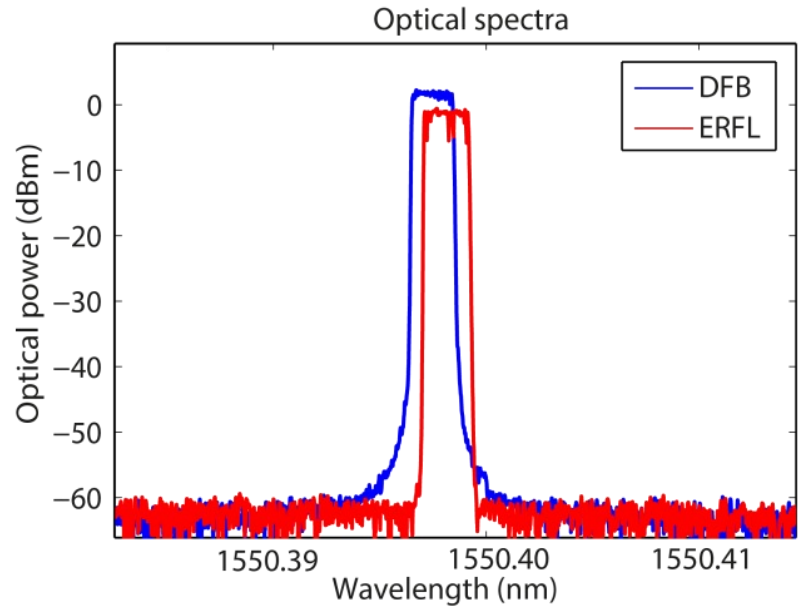

b)

Figure 2. Spectral comparison between DFB laser and ERFL. a) Optical output spectra of DFB laser (blue line) and ERFL (red line). b) Wavelength stability after some minutes of DFB laser (blue line) and ERFL (red line).

To check if this kind of laser based on Erbium doped fiber could be used as a laser source of a BOTDA system, a distributed measurement has been carried out. As sensing fiber a uniform $21 \mathrm{~km}$ long single mode fiber at room temperature has been employed, where the last $15 \mathrm{~m}$ have been placed inside a climatic chamber at $50{ }^{\circ} \mathrm{C}$ to measure a hot spot. The spatial resolution of the sensor was fixed to $2 \mathrm{~m}$ and the frequency of the probe wave was swept from 10.6 $\mathrm{GHz}$ to $10.9 \mathrm{GHz}$ with a frequency step of $2 \mathrm{MHz}$. As previously, the output power of both lasers has been reduced to OdBm with an optical attenuator.

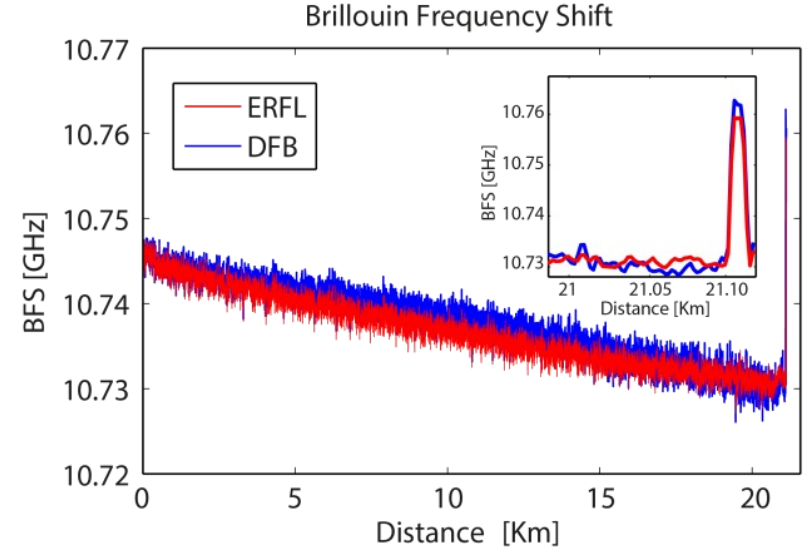

a)

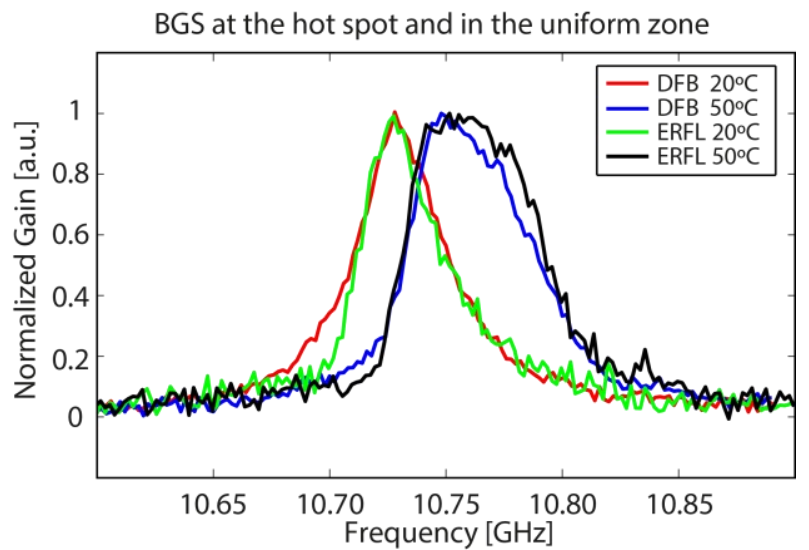

b)

Figure 3. Distributed measurement using BOTDA sensor system and DFB laser (blue line) and ERFL (red line). a) BFS estimation applying Lorentzian fitting of the acquired BOTDA traces over a $21 \mathrm{~km}$ long fiber where the last $15 \mathrm{~m}$ were heated at $50{ }^{\circ} \mathrm{C}$. The inset shows a zoom view of the last $100 \mathrm{~m}$ of the fiber. b) BGS acquired near the end of the sensing fiber, at the hot spot section and some meters before with DFB laser (blue and red line respectively) and with the ERFL (black and green line respectively). 
The BFS estimated after using Lorentzian fitting over the BGS measured with the Brillouin sensor along the $21 \mathrm{~km}$ fiber is represented in Figure 3 (a). As can be observed, the distribution of the BFS is practically the same for both lasers, DFB and ERFL (blue and red line respectively). An inset has also been included in the figure to verify that the hot spot is correctly resolved, showing a difference of about $30 \mathrm{MHz}$, from 10.73 to $10.76 \mathrm{GHz}$, corresponding to a temperature change of about $30^{\circ} \mathrm{C}$. Figure 3 (b) shows the normalized BGS at the hot spot and at the room temperature sections. Comparing the BGS for both lasers, it is appreciable that the noise seems to be a little higher, especially at the frequencies where the Brillouin gain is lower, when the laser employed is the ERFL (green and black lines) instead of using the DFB laser (red and blue lines). But the shapes and the peak values of the BGS are practically the same. Figure 3 evidence that an ERFL could be used as the laser source of a BOTDA sensor to performance distributed measurements with similar results than using a DFB laser diode.

\section{CONCLUSIONS}

In this work, we have studied the feasibility of employing a fiber laser as a laser source of a BOTDA sensor to perform distributed measurements based on Brillouin scattering. The designed fiber laser is an Erbium Fiber Ring Laser structure working on the Single Longitudinal Mode regime. To analyze the viability of this approach for BOTDA systems, a hot spot at $50^{\circ} \mathrm{C}$ has been measured at the end of a $21 \mathrm{~km}$ fiber and the associated results have been compared with those of a commercial DFB laser. Moreover, some key laser parameters like the output spectra and the wavelength stability have been measured and also compared with the DFB. The performance obtained with the ERFL on BOTDA sensors shows the viability of use fiber laser on experimental setups. Among other possible advantages, the ability of the proposed structure to be tuned over a wide spectral range might prove interesting within this framework.

\section{ACKNOWLEDGEMENTS}

This work has been supported by the projects TEC2013-47264-C2-1-R and TEC2016-76021-C2-2-R.

\section{REFERENCES}

[1] Angulo-Vinuesa, X., Martin-Lopez, S., Nuño, J., Corredera, P., Ania-Castañon, J. D., Thévenaz, L., and González-Herráez, M., "Raman-assisted Brillouin distributed temperature sensor over $100 \mathrm{~km}$ featuring $2 \mathrm{~m}$ resolution and 1.2 C uncertainty," Journal of Lightwave Technology, 30(8), 1060-1065 (2012).

[2] Urricelqui, J., Sagues, M., and Loayssa, A., "Brillouin distributed sensing assisted by Brillouin amplification of pump pulses." IEEE SENSORS 2014 Proceedings,1776-1779 (2014).

[3] Li, W., Bao, X., Li, Y., and Chen, L., "Differential pulse-width pair BOTDA for high spatial resolution sensing," Optics express, 16(26), 21616-21625 (2008).

[4] Bernini, R., Minardo, A., and Zeni, L., "Dynamic strain measurement in optical fibers by stimulated Brillouin scattering," Optics letters, 34(17), 2613-2615 (2009).

[5] Soto, M. A., and Thévenaz, L., "Modeling and evaluating the performance of Brillouin distributed optical fiber sensors," Optics express, 21(25), 31347-31366 (2013).

[6] Urricelqui, J., Soto, M. A., and Thévenaz, L., "Sources of noise in Brillouin optical time-domain analyzers." International Conference on Optical Fibre Sensors (OFS24), 963434-963434-4 (2015).

[7] Shlomovits, O., Langer, T., and Tur, M., "The effect of source phase noise on stimulated brillouin amplification,” Journal of Lightwave Technology, 33(12), 2639-2645 (2015).

[8] Minardo, A., Bernini, R., and Zeni, L., "Analysis of SNR penalty in Brillouin optical time-domain analysis sensors induced by laser source phase noise," Journal of Optics, 18(2), 025601 (2015).

[9] Liu, J., Yao, J., Yao, J., and Yeap, T. H., "Single longitudinal mode multi-wavelength fiber ring lasers." Optical Fiber Communication Conference, (2004). 